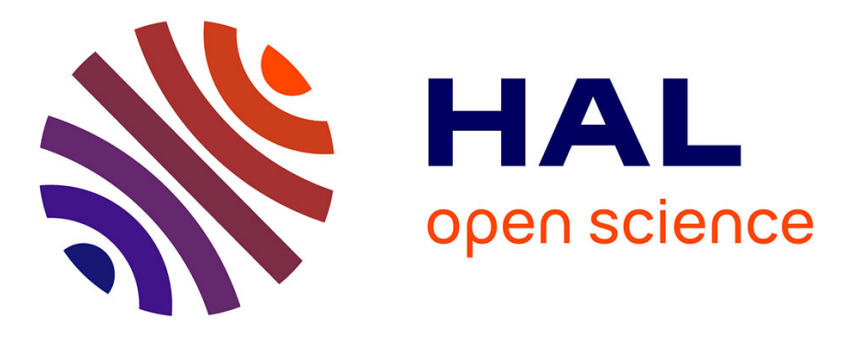

\title{
Les maisons antiques de la rue des Magnans à Aix-en-Provence (Bouches-du-Rhône)
}

Géraldine Bérard, Brigitte de Luca, Corinne Landuré, Andréas Nicolaïdès

\section{To cite this version:}

Géraldine Bérard, Brigitte de Luca, Corinne Landuré, Andréas Nicolaïdès. Les maisons antiques de la rue des Magnans à Aix-en-Provence (Bouches-du-Rhône). Gallia - Fouilles et monuments archéologiques en France métropolitaine, 1994, 51, pp.191-201. 10.3406/galia.1994.2977. hal01912102

\section{HAL Id: hal-01912102 \\ https://hal.science/hal-01912102}

Submitted on 29 Jan 2020

HAL is a multi-disciplinary open access archive for the deposit and dissemination of scientific research documents, whether they are published or not. The documents may come from teaching and research institutions in France or abroad, or from public or private research centers.
L'archive ouverte pluridisciplinaire HAL, est destinée au dépôt et à la diffusion de documents scientifiques de niveau recherche, publiés ou non, émanant des établissements d'enseignement et de recherche français ou étrangers, des laboratoires publics ou privés.

\section{(ㅇ)(1) $\$$}

Distributed under a Creative Commons Attribution - NonCommercial - NoDerivatives| 4.0 


\section{Les maisons antiques de la rue des Magnans à Aix-en-Provence (Bouches-du-Rhône)}

\section{par Géraldine BÉRARD ${ }^{1}$, Brigitte DE LUCA ${ }^{2}$, Corinne LANDURÉ 3 et Andréas NICOLAÏDÈS4}

(19) Une fouille réalisée au 18 rue des Magnans à Aix-en-Provence a permis la mise au jour de vestiges d'habitations datés du II s. de notre ère. situés non loin du firum. Deux corps de bâtiment. incomplètement dégagés. sont établis de ipart et d'autre d'un passage, sur un terrain aménagé en terrasses. Parmi les découvertes notables se distinguent. dans le triclinium, une mosäque polychrome particulièrement bien conservée, ainsi que des peintures murales en place.

An emergency excaration at 18 , rue des Magnans, Air-en-Provence, brought to light vestiges of a settlement dating from the 2 ind century A.D. located in the vicinity of the forum. Two building structures partly excarated were found on either side of a passage on terraced ground. Among the most remarkable finds is a particularly well preserved polychrome mosaic, decorating the triclinium floor. In the triclinium we also discolered frescoes remaining on the lower part of the walls.

Mots clés : Bouches-du-Rhône, Aix-en-Provence, habitations urbaines, gallo-romain. II s., puits, peintures murales. mosaïque.

I AFAN. Antenne Méditerranée.

2 AFAN. Antenne Méditerranéc.

3 Service régional de l'Archéologie de Franche-Comté.

4 Chercheur associć au Laboratoirc d'Archéologic Médiévale Méditerranéenne UMR 9905. CNRS. Gallia. 51. 1994, p. 191-201. 
A la suite d'un projet immobilier, une fouille archéologique conduite sur une parcelle de $250 \mathrm{~m}^{2}$ située dans le centre d'Aix-en-Provence, au 18 rue des Magnans ${ }^{5}$ (fig. 1), a permis la mise au jour d'habitations qui appartiennent à deux phases d'occupation distinctes : le Moyen Age (XIII ${ }^{e}-X^{2}{ }^{e}$ s.), et l'Antiquité ( $\mathrm{II}^{\mathrm{e}} \mathrm{s}$.), seule période étudiée dans le présent article.

Le site des Magnans s'inscrit dans la partie sud-est de l'agglomération romaine, à proximité du tracé de la limite sud de la ville tel qu'il est défini de manière hypothétique par R. Ambard (Ambard, 1984, p. 65-71). Il est proche également du forum et de ses dépendances (Fixot et alii, 1986), ainsi que de l'ensemble monumental révélé par les fouilles des Thermes (fig. 2).

Dans ce secteur de la ville, l'habitat antique n'était véritablement connu qu'à travers les fouilles de la cour de l'archevêché où des insulae furent découvertes près du forum (Fixot et alii, 1986), même si quelques opérations de moindre ampleur avaient également livré des vestiges plus fragmentaires d'habitations, notamment sous la cathédrale, la rue de la Louvière (Nin, 1990, p. 136-137), la Maison d'Arrêt ${ }^{6}$ et la rue Lice des Cordeliers (Ambard, 1984, p. 148).

Les fouilles de la rue des Magnans offraient donc l'occasion de mieux appréhender l'urbanisation de cette partie de la ville, qui, on le voit, restait encore assez peu explorée.

Les vestiges identifiés sont implantés sur deux terrasses dont la création fut dictée par le fort pendage est-ouest du terrain, encore visible aujourd'hui dans la topographie des rues voisines. Ceux-ci appartiennent à deux bâtiments imparfaitement reconnus situés de part et d'autre d'un espace large de $4.70 \mathrm{~m}$. l'espace I, qu'il faut sans doute interpréter comme une zone de passage (fig. 3 et 4). De direction générale est-nord-est/ouestsud-ouest, ces constructions adoptent un axe sensiblement différent.

5 La fouille de sauvetage urgent s'est dérouléc du mois d'octobre au mois de décembre 1990, à la suite d'une campagne de sondages préliminaires réalisćc par M. Bonifay du 30 août au 4 septembre 1990 (Bonifay, 1990). Le dégagement des vestiges, pratiqué de manière exhaustive sur la moitić est seulement du terrain. a été parallèlement étendu sur $15 \mathrm{~m}^{2}$ dans la rue des Magnans pour effectuer la dépose partielle de la mosaique polychrome. En février et en mars 1992. A. Nicolaïdès eut la possibilité d'agrandir ce sondage el de cerner ainsi l'emprise du pavement dont la dépose put être achevée (Nicolaïdès, 1992).

6 De nombreuses informations sont issues du Bilan scientifique. Celui-ci n’étant pas considéré, à proprement parlé. comme une publication, nous ne faisons ici qu'une mention générale : Bilan scientifique. DRAC Provence-AlpesCôte d'Azur, 1992, p. 101-113.

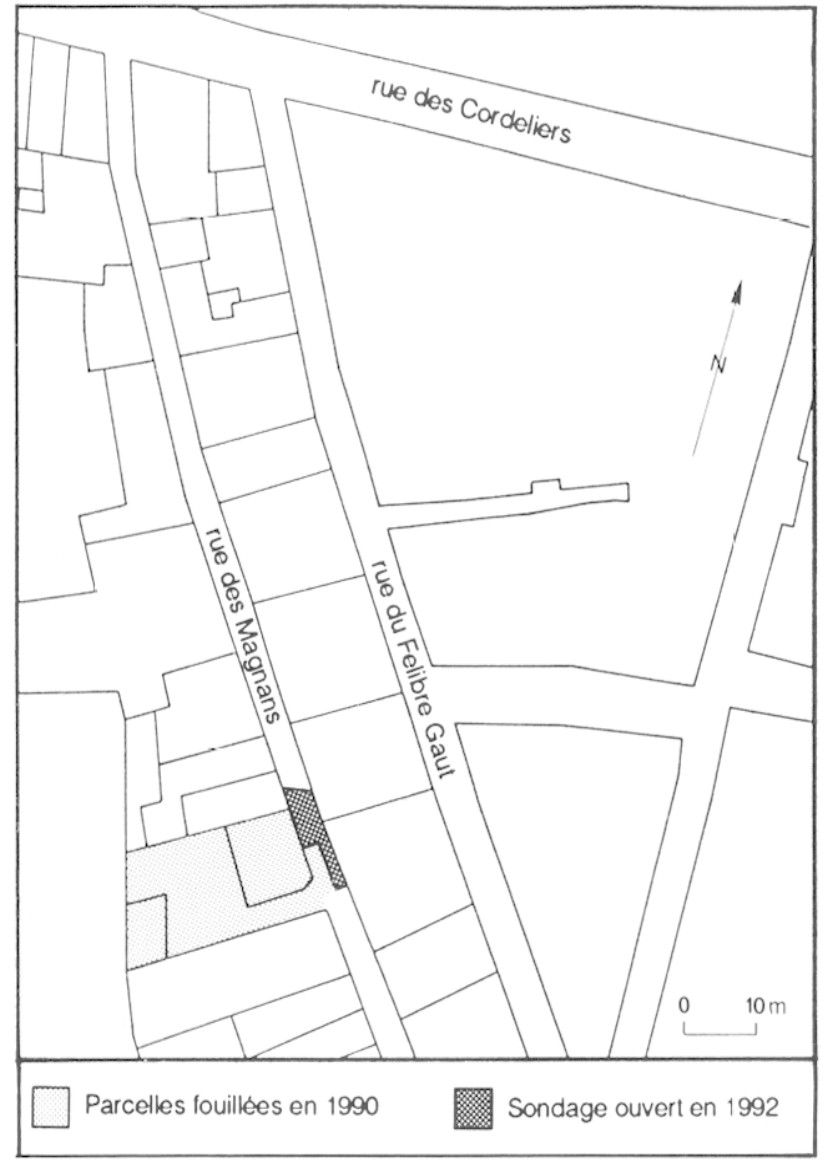

Fig. 1 - Situation de la fouille sur fond de cadastre actuel.

\section{LA TERRASSE INFÉRIEURE}

Épargnée par le projet immobilier, la terrasse inférieure, située $1.50 \mathrm{~m}$ en contrebas de l'espace I, n'a pas donné lieu à une recherche très poussée. A la faveur de deux sondages et d'une surveillance de tranchée, nous avons pu néanmoins dresser le plan incomplet de quatre pièces ou espaces ( $J, K, L$ et $N$ ) adossés à l'est, pour deux d'entre eux au moins, au mur de soutènement $M$ XIII, seule limite atteinte du corps de bâtiment ouest.

La date d'implantation et l'évolution éventuelle de ces constructions nous échappent également, car un seul état doccupation, scellé par une couche de destruction, a été identifié. Il n'a pas été possible en effet de vérifier l'existence éventuelle de niveaux antérieurs sous les sols dégagés, que nous avons dû laisser en place.

L'absence de matériel significatif dans les sondages n'a pas permis en outre de définir les périodes d'utilisation et d'abandon de ces aménagements. 


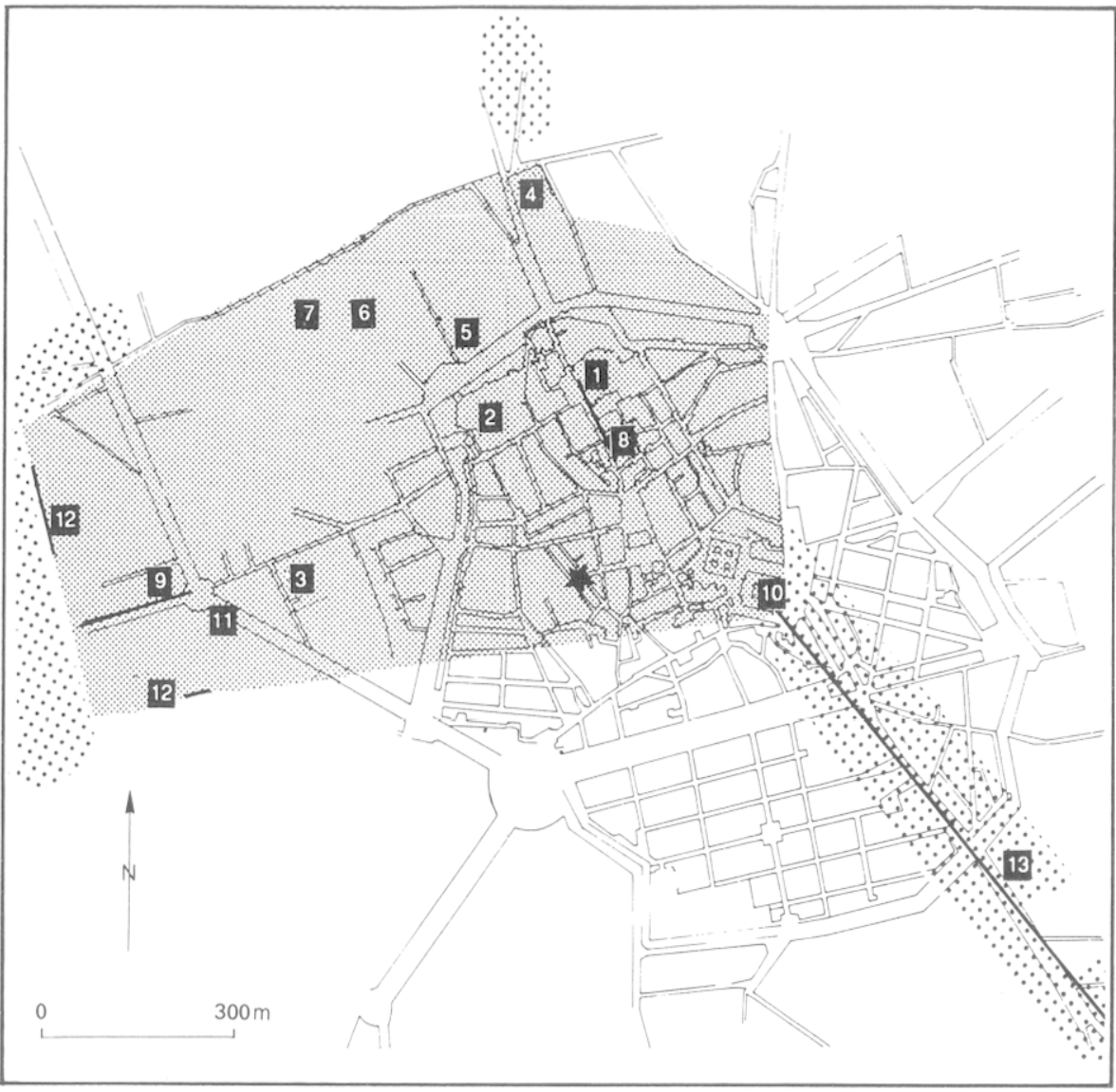

Fig. 2 - Plan schématique d'Aix Antique.

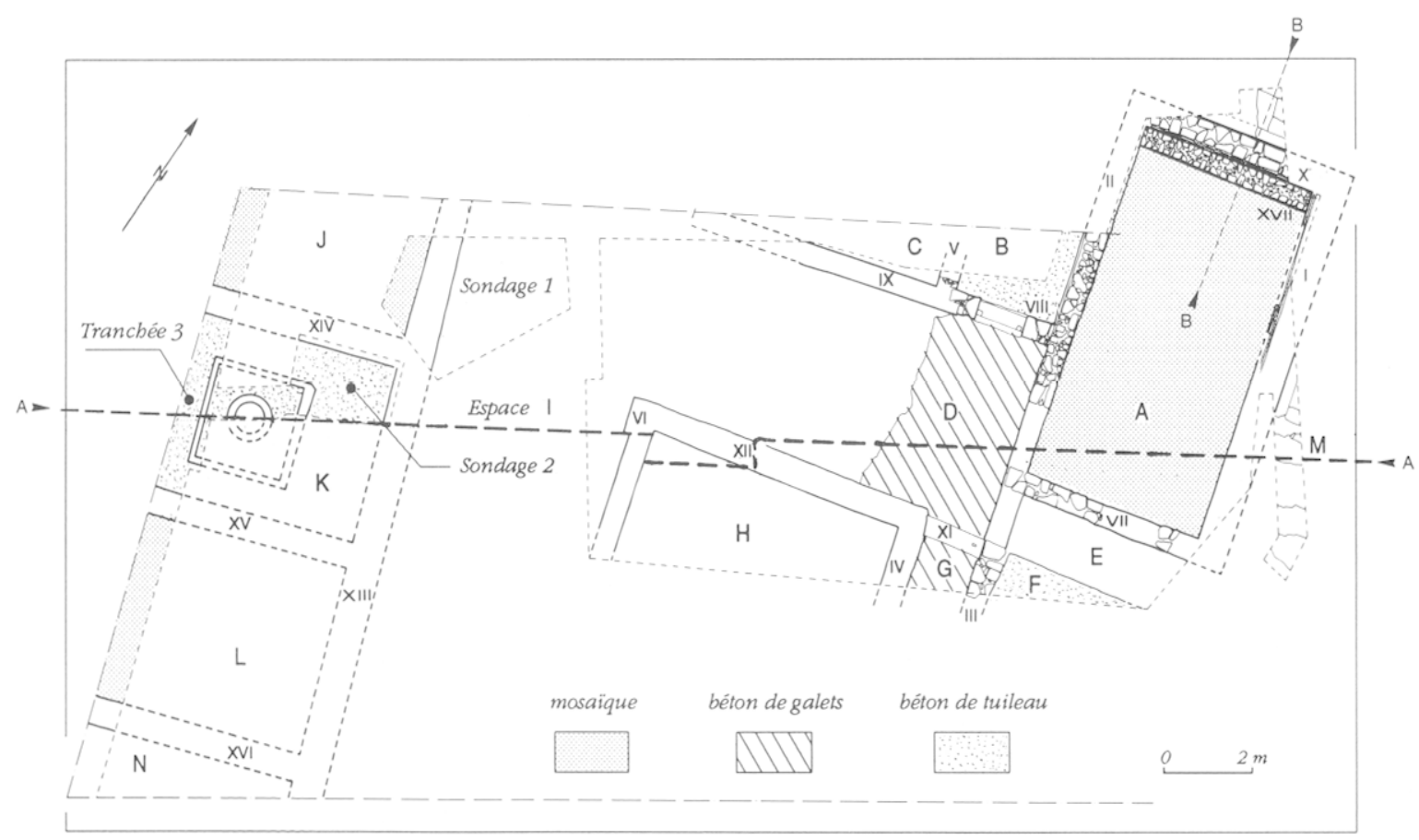

Fig. 3 - Plan des vestiges antiques. 


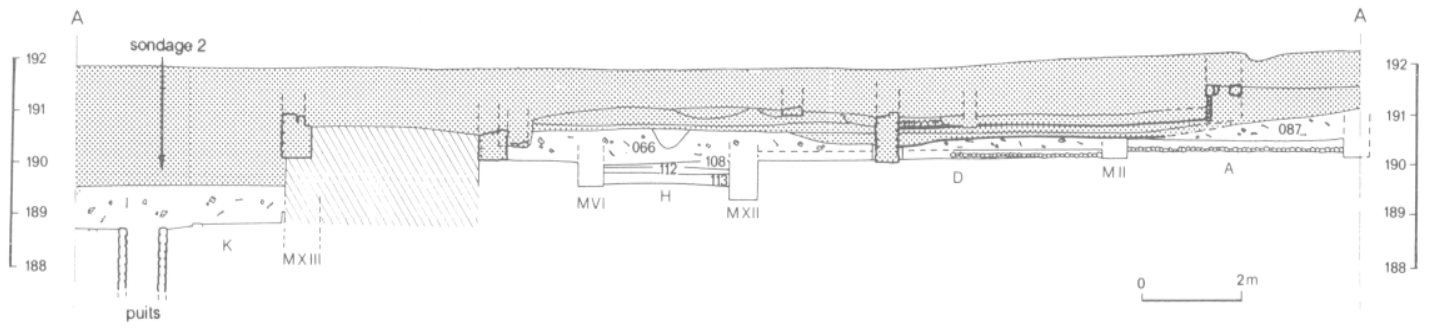

Fig. 4 - Coupe stratigraphique A-A'. En grisé : les niveaux d'époque médiévale.

\section{Description des vestiges}

L'espace le mieux appréhendé est l'espace $\mathrm{K}$, d'une superficie minimale de $17.20 \mathrm{~m}^{2}$. Son sol, en béton de tuileau, présente de légères pentes ${ }^{7}$ qui convergent vers un puits, autour duquel une bordure de pierres calcaires blanches dessine une figure presque carrée $(2.40 \mathrm{~m} \mathrm{x}$ $2.30 \mathrm{~m}$ ), un peu décentrée par rapport aux limites sud et nord de la pièce (fig. .5) $)^{8}$.

La margelle du puits ${ }^{9}$, d'un diamètre hors-ouvre de $0.67 \mathrm{~m}$, est construite avec deux blocs calcaires taillés en arc de cercle tandis que ses parois internes sont montées en moellons équarris disposés en assises assez régulières liées à la terre. La profondeur de l'ouvrage reste indéterminée et nous ignorons s'il atteignait ou non la nappe phréatique.

Le puits et sa bordure, réalisés avec des matériaux de qualité mis en ceuvre avec soin. constituent une belle construction qu'il convient sans doute de replacer dans un espace dépourvu de toiture. Bien que l'implantation du puits dans un lieu couvert ne soit pas à exclure - on rencontre un tel exemple dans le péristyle d'une des maisons du jardin de Grassi à Aix-en-Provence (Benoit, 1947. p. 110) - l'aménagement en pente du sol de l'espace K suscite en effet cette interprétation et il paraît vraisemblable que nous soyons ici dans une cour où l'on a dirigé le ruissellement des eaux pluviales vers le puits, de sorte qu'elles ne s'infiltrent ni ne pénètrent dans les pièces $\mathrm{J}$ et $\mathrm{L}$ mitoyennes au nord et au sud.

Le sol des pièces $\mathrm{J}$ et $\mathrm{L}^{10}$, à peine entrevu, est un pavement de mosaïque blanche auquel répondait, dans la

7 Cote $188.89 \mathrm{~m} \mathrm{NGF}$ contre le mur XIII. $188.85 \mathrm{~m} \mathrm{NGF}$ à $1.60 \mathrm{~m}$ de ce point. $188.81 \mathrm{~m} \mathrm{NGF}$ contre la bordure de pierres et $188.75 \mathrm{~m} \mathrm{NGF}$ contre la margelle du puits.

8 Cette bordure montre des largeurs inégales variant de 0.13 à $0.24 \mathrm{~m}$ selon les côtés, elle affleure à la surface du sol de circulation mais est à peine surélevée $(0.04 \mathrm{~m})$ par rapport au sol de l'espace central. Elle se situe à $0,70 \mathrm{~m}$ du mur XV et à $0.80 \mathrm{~m}$ du mur XIV.

9 Qui est à peine surélevée par rapport au sol $(0.02 \mathrm{~m})$.

10 Le sol de ces pièces se situe à la cote 188,85 m NGF. pièce $\mathrm{L}$ au moins, un décor mural de qualité ainsi qu'en témoignent de nombreux fragments d'enduits peints, colorés avec du rouge cinabre ou du bleu égyptien, retrouvés dans le niveau de destruction de cette pièce (Boiron et alii, 1986, p. 35).

La pièce voisine $\mathrm{N}$ devait être plus sommaire si l'on en juge par la nature de son sol, en simple terre battue.

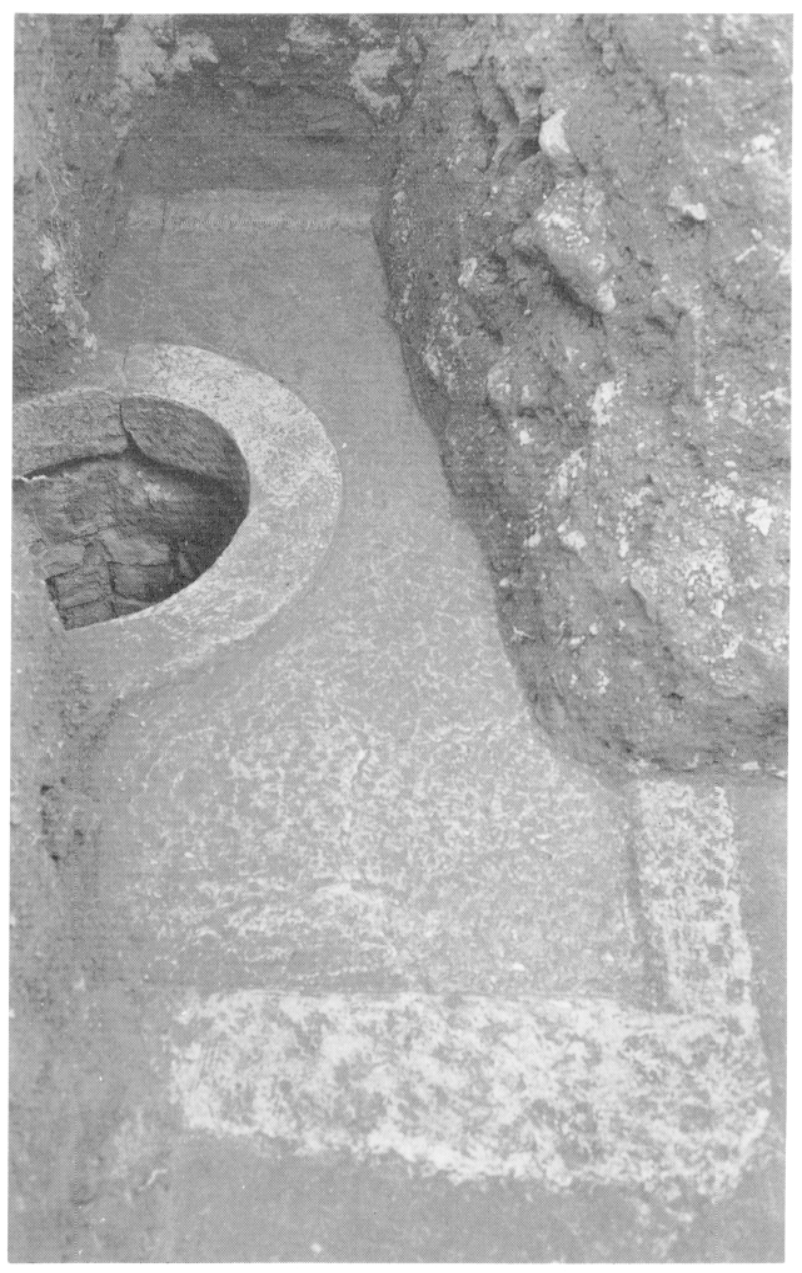

Fig. 5 - Puits de la pièce K. 


\section{LA TERRASSE SUPÉRIEURE}

Les bâtiments découverts sur la terrasse supérieure. explorée sur $120 \mathrm{~m}^{2}$, appartiennent à une aile d'habitation dont seule la limite ouest est identifiée avec certitude. Large au moins de $14 \mathrm{~m}$, elle comporte au minimum 9 pièces et espaces, dont deux seulement. l'espace D et la pièce A, ont pu être totalement restitués (fig. 3 et 6 ).

Hormis une petite réparation du sol du couloir $\mathrm{G}$ et la construction d'une banquette maçonnée (XVII) dans la pièce $A$. les différents espaces dégagés ne semblent pas avoir subi d'importants remaniements, visant, par exemple. à modifier l'agencement interne, ou. phénomène plus courant, à rénover les sols et les enduits muraux. Aucun d'entre eux n'a livré en effet plus d'un niveau d'occupation, les sols étant installés directement sur le substrat ou sur des remblais de nivellement.

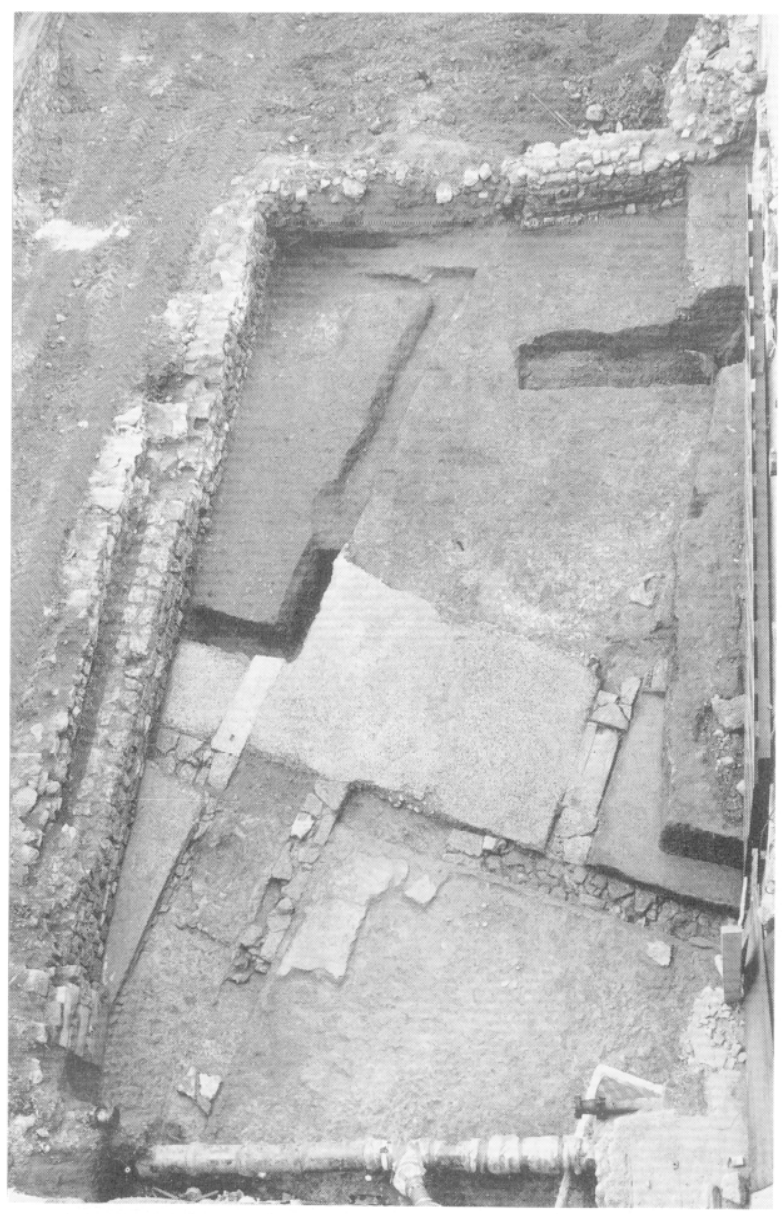

Fig. 6 - Vue d'ensemble de la terrasse supérieure prise depuis la rue des Magnans.

\section{L'espace I : une rue ?}

Établi en limite ouest de la terrasse supérieure, l'espace I a été très partiellement fouillé dans le sondage 1 (fig. 3). Cependant, sa situation entre les bâtiments des terrasses inférieure et supérieure, ainsi que l'aspect de son sol. un remblai de safre remanié à la surface légèrement damée et caillouteuse, nous incitent à l'interpréter comme une zone de passage, qu'il serait plus tentant, vu ses dimensions $(4,70 \mathrm{~m}$ de largeur), d'assimiler à une rue plutôt qu'à un passage privé servant d'articulation aux deux corps de bâtiment.

\section{Description des vestiges}

\section{Les murs}

Seuls les murs de la partie orientale du bâtiment ont été en partie préservés, mais la plupart ne subsistent pratiquement plus qu'en fondations (M II, III, V. VII et VIII), qu'accompagnent parfois des vestiges ténus de plinthes d'enduits peints (M II. VII et VIII).

Ces fondations présentent les mêmes caractéristiques : très peu profondes "I, elles reposent sur un substrat de marnes grises et sont constituées de pierres calcaires blanches de dimensions moyennes, extraites en plaque et liées à l'argile. Leur largeur varie de $0.45 \mathrm{~m}$ à $0.60 \mathrm{~m}$.

Les murs de la pièce $\mathrm{A}$ ont conservé en revanche une partie de leur élévation. Le mur X, entièrement reconnu. est visible sur une hauteur maximale de $0,66 \mathrm{~m}$, alors que le mur I. identifié sur $4 \mathrm{~m}$ de longueur et récupéré dans sa portion sud, a été observé sur $0.70 \mathrm{~m}$ de hauteur ${ }^{12}$. Bâtis de façon similaire, ils sont connstilués de deux parements de gros moellons équarris montés en appareil soignél 13 et ont fait l'objet. du côté de la pièce, d'un traitement spécifique. Contre leur parement a été appliqué une première couche de mortier de $2 \mathrm{~cm}$ d'épaisseur, brossée en surface, dans laquelle ont été fichés des crampons de fer en forme de $T$ servant de scellement à des tegulae jointives, disposées dans le sens de la hauteur. La face externe de ces tuiles a été enduite d'une autre couche de mortier un peu plus grossier que recouvrait enfin une dernière couche de mortier de chaux, de texture très fine, utilisée comme

11 La profondeur des fondations est de $0.15 \mathrm{~m}$ pour le mur II. $0.21 \mathrm{~m}$ pour le mur V. $0.05 \mathrm{~m}$ pour le mur VII et $0.20 \mathrm{~m}$ pour le mur VIII. $\mathrm{MX}$.

12 Leur largeur est de $0.54 \mathrm{~m}$ pour M I et de $0.45 \mathrm{~m}$ pour

1.3 Les moellons, de taille variable $(0.30$ à $0.40 \mathrm{~m}$ de long : 0.15 à $0.20 \mathrm{~m}$ de haut : $0.20 \mathrm{~m}$ de large en moyenne). sont disposés en assises régulières et liés avec un mortier de chaux blanchâtre, riche en particules grossières (petits galets). Le blocage interne des murs est fait de petites pierres retaillées également liées au mortier de chaux. 


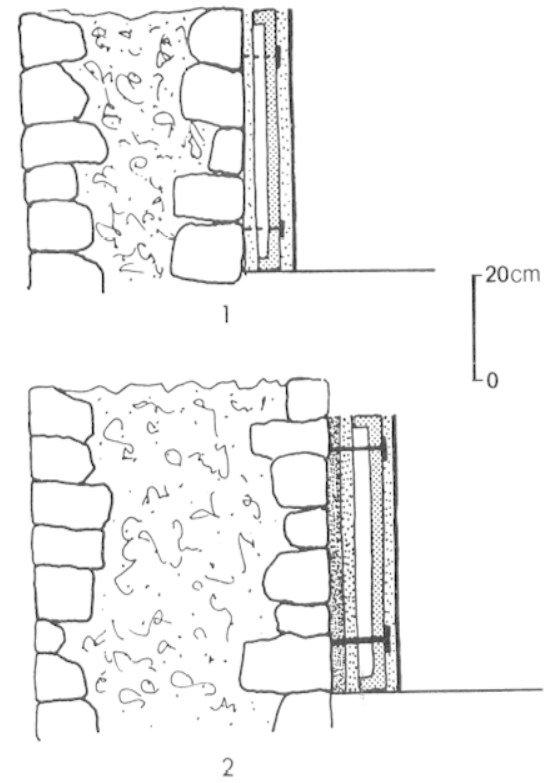

Fig. 7 - Coupes nord-sud du mur $X\left(n^{\circ} 1\right)$ et est-ouest du mur I $\left(n^{\circ} 2\right)$.

support de la peinture murale (fïg. 7, $\mathrm{n}^{\circ} 1$ ). Le revêtement du mur I est à peine différent de celui du mur X : au contact du parement a été observé une couche supplémentaire de mortier de chaux enrichie de charbons de bois (fig. 7, $n^{\circ} 2$ ).

De tels dispositifs, qui atteignent des épaisseurs relativement importantes $(0,10 \mathrm{~m}$ pour $\mathrm{MX}$ et $0.18 \mathrm{~m}$ pour $M$ I), ne correspondent visiblement pas à un système de chauffage mural puisque le sol de la pièce $A$ n'est pas construit sur hypocauste. Ils témoignent en fait d'un souci d'isolation et répondent aux recommandations prescrites par Vitruve dans son livre VII du $D e$ Architectura (chapitre II-IV, 3, p. 87-101) pour pallier les problèmes d'humidité rencontrés dans les locaux exposés.

Il est possible, dans le cas présent, que les constructeurs aient eu à prévoir dès l'origine, ou à résoudre à l'usage. des problèmes d'infiltration d'eaux que pouvait en effet entraîner l'importante différence de niveau existant entre le sol de la pièce $A$, et celui, établi plus haut, des espaces mitoyens situés au nord et à l'est.

Les murs de la partie ouest du bâtiment, totalement spoliés, ne se prêtent à aucune observation. Tout au plus peut-on constater qu'ils étaient plus profondément fondés que les autres ${ }^{14}$. sans doute en raison de la nature sableuse du substrat à cet endroit.

14 La profondeur des fondations est de $0.33 \mathrm{~m}$ pour le mur IX. $0.45 \mathrm{~m}$ pour le mur VI. $0.57 \mathrm{~m}$ pour le mur IV et $0.91 \mathrm{~m}$ pour le mur XII.

\section{Les pièces et espaces}

Le bâti reconnu s'organise autour d'un espace central de plan rectangulaire $(4 \mathrm{~m} \times 8 \mathrm{~m})$. l'espace $D$. où est encore présent, sur $3 \mathrm{~m}$ de profondeur, un sol de béton de galets assez grossier ${ }^{15}$ disposé sur un radier de pierres ${ }^{16}$.

Cet espace jouait de toute évidence un rôle essentiel dans la distribution des pièces attenantes puisqu'il donnait accès au moins à deux d'entre elless, la pièce B et le couloir G, dont les pierres de seuil subsistent, ainsi. sans doute. qu'à la pièce $A$ et au couloir $\mathrm{E}$.

L'absence de toute trace de mur de fermeture occidental semble indiquer qu'il était totalement ouvert sur l'espace $1^{17}$, mais nous ignorons en revanche s'il s'agissait d'un vestibule couvert, ou d'une petite cour qui pouvait apporter lumière et aération aux pièces voisines.

La circulation à l'intérieur de l'aile d'habitation se faisait par l'intermédiaire des couloirs $E$ et $\mathrm{G}^{18}$ qui devaient probablement se poursuivre dans les parties non atteintes de la maison.

Le couloir $\mathrm{G}$, large de $1,30 \mathrm{~m}$, a été identifié sur $1.40 \mathrm{~m}$ de longueur entre les pièces $\mathrm{H}$ et $\mathrm{F}$. Il débouchait sur l'espace D par une ouverture matérialisée au sol par un bloc taillé de calcaire blanc, dépourvu de feuillures et vraisemblablement en réemploi ${ }^{19}$ (fig. 8). Son sol, qui comporte une petite réparation réalisée en béton de tuileau, est identique à celui de l'espace D.

Implanté entre les pièces A et F, le couloir E. à peine plus étroit $(1.10 \mathrm{~m})$. devait logiquement communiquer lui aussi avec l'espace central. Il ne reste plus de son sol qu'une bande d'emprise réduite (moins de $1 \mathrm{~m}^{2}$ ) formée d'un lit de tuiles brisées et de pierres liéess au mortier. Sans doute s'agit-il d'un vestige de radier ayant servi de base à un sol en dur.

15 Le sol de la pièce D se situe à la cote 190.36 m NGF. de même que celui de l'espace $\mathrm{G}$.

16 Tous les sols maçonnés de la terrasse supérieure reposent directement sur le substrat, le pendage du terrain étant compensé par le soubassement des sols cux-mêmes dont la hauteur varie.

17 C'est ce que suggère en tout cas l'absence de tranchée de récupération d'un éventuel mur de fermeture dont la disparition serait étonnante si l'on considere la profondeur importante des tranchées voisines (M VI et M XII).

18 Pour l'espace E. très mal conservé. l'éventualité d'un escalier semble à exclure : aucune trace d'étage effondré n'a été décelée à la fouille el aucun mur porteur n'est présent au sud de l'espace.

$19 \mathrm{Ce}$ bloc (longueur: $1.45 \mathrm{~m}$ : largeur : $0.45 \mathrm{~m}$ : hatuteur : $0.19 \mathrm{~m}$ à l'est et $0.22 \mathrm{~m}$ à l'ouest) est cassé sur le côté ouest où il pénètre légèrement dans l'axe de récupération des murs IV et XII. Il comporte à son extrémité orientale un trou rectangulaire délicat à interpréter. empreinte de crapaudine ou trace d'ancrage de support. dont il est difficile de savoir s'il était lié ou non à l'utilisation en réemploi du bloc. 

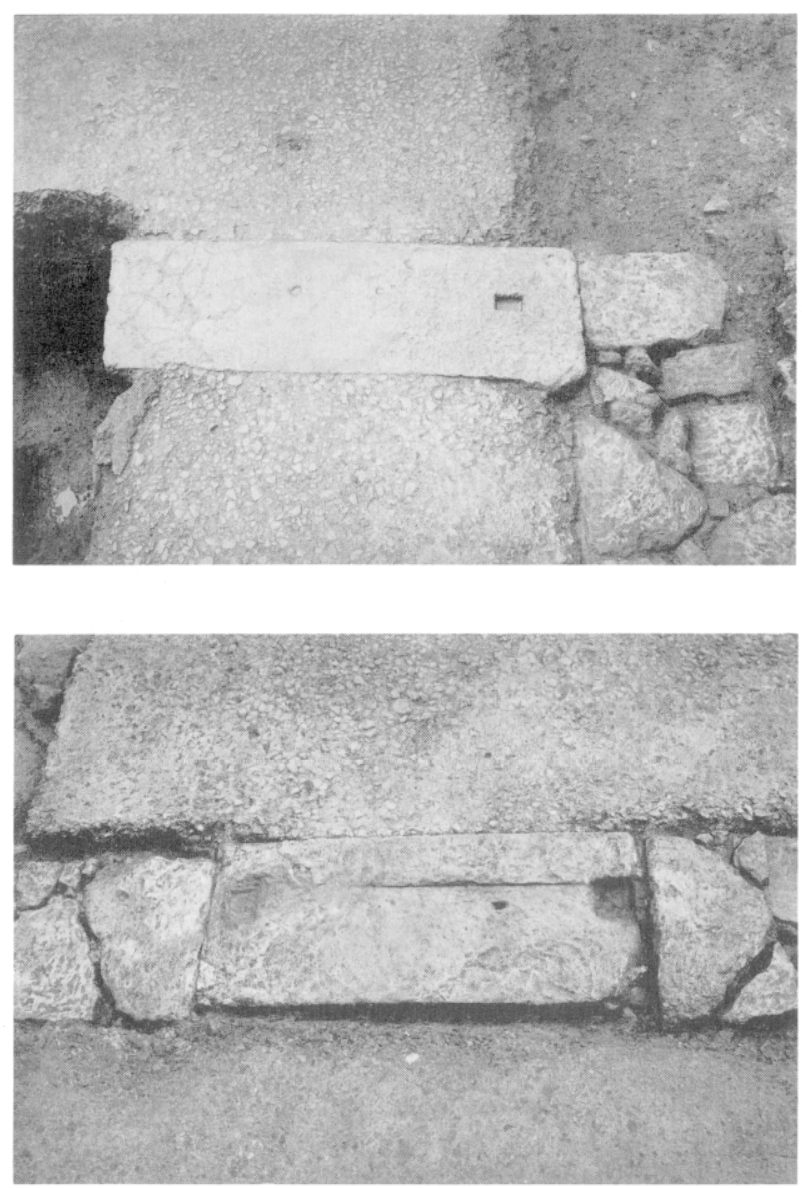

Le mur VII délimitait le passage au nord tandis qu'une simple cloison venait semble-t-il le fermer du côté sud. Le long de la bordure bien lissée du béton de la pièce $F$ courait en effet une fine tranchée $(0,10 \mathrm{~m}$ de largeur) comblée de fragments de tuiles et de petites pierres bien agencées, aménagement qui pourrait correspondre au soubassement d'une mince paroi.

Les pièces $B, C$ et $F$, toutes pourvues de béton de tuileau, ont été très peu dégagées.

La pièce $\mathrm{B}^{20}$. qui mesure $2,40 \mathrm{~m}$ d'est en ouest, devait être de petite taille. Elle donnait sur l'espace D par une ouverture bien centrée de 1,12 m, dont la porte, à double battant, s'ouvrait vers l'intérieur de la pièce. C'est ce que laisse observer son seuil monolithe de calcaire blanc qui comporte deux vestiges de crapaudines ainsi qu'une feuillure (fig. 9).

Aucun aménagement comparable n'a été retrouvé dans les deux autres pièces dont nous ignorons les dimensions. Seule pourrait être hypothétiquement replacée la limite ouest de la pièce $\mathrm{C}$, dans l'axe de celle de la pièce $H$.

20 Le sol de la pièce B se situe à la cote 190 m NGF.
Fig. 8-Bloc de pierre calcaire $\mathrm{MXI}$. vue prise depuis le sud.

Fig. 9-Seuil d'accès à la piece $B$, vue prise depuis le sud.

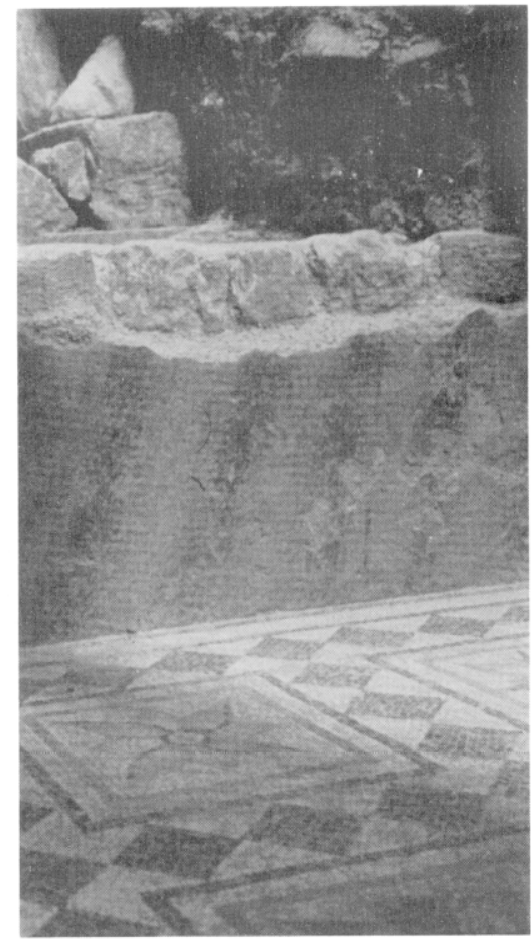

Fig. $10-$ Vue de la banquette.

Nous ne connaissons que la dimension est-ouest de cette pièce $(6 \mathrm{~m})$, qui se développe sur au moins $3 \mathrm{~m}$ du nord au sud et se distingue par la nature de son sol, en terre battue 21 .

Tel n'est pas le cas de la pièce $A$, ornée d'un très beau pavement de mosaïque polychrome 22 , qu'une banquette maçonnée, établie du côté nord le long du mur $X$, dissimule très partiellement (fïg. 10). La salle, de plan rectangulaire $(8 \mathrm{~m} \times 4 \mathrm{~m})$, ne disposait donc pas à l'origine de cet aménagement, seul témoin notable de l'existence d'une campagne de travaux durant l'occupation du bâtiment, du moins dans les parties reconnues de celui-ci. Le mur $X$ fut en effet doublé sur toute sa longueur par la banquette XVII, large de $0,46 \mathrm{~m}$ et haute de $0,50 \mathrm{~m}$ (fig. 11 ). Construite sur la mosaïque et entièrement revêtue d'un enduit peint, celleci est bâtie en petits moellons taillés bien appareillés, liès par un mortier de chaux rose. La couche de mortier assez grossier (2,3 cm d'épaisseur) qui en recouvrait les faces visibles avait été peint a fresco. Du décor de l'assise, endommagé, on ne discerne plus qu'une courte ligne de festons de couleur ocre-rouge, à proximité du mur X. Celui du parement (fig. 12, p. 201), beaucoup mieux conservé, montre une alternance de bandes obliques, peintes en dégradé dans les tons bleu clair à

21 Le sol de la pièce $H$. situé à la cote 190,05 m NGF. recouvrait deux remblais préparatoires de nivellement.

22 L'étude de la mosaïque polychrome de la pièce A a été réalisée par H. Lavagne, voir infica, p. 202-215. 


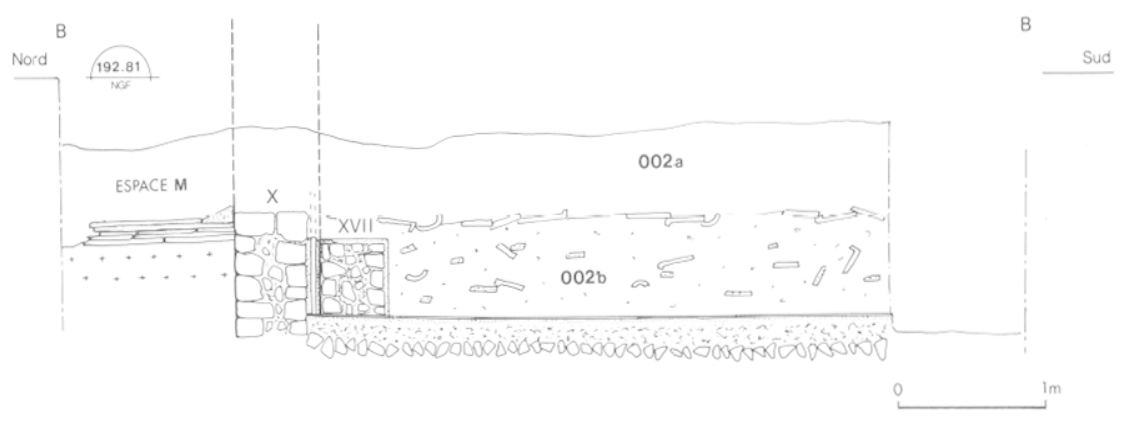

Fig. 11 - Coupe B-B` (cf. lig. 3) de la zone de fouille de 1992.

bleu foncé et obtenues par larges coups de pinceau dont les traces sont encore visibles par endroits.

Ce traitement pictural, qui imite les placages de marbre. est en fait un décor asséz courant ${ }^{23}$. Dans le cas présent, deux filets noirs moins obliques que les bandes imitant le marbre ont été peints a secroo par-dessus.

La présence de la banquette, disposée dans le sens de lecture du pavement mosaïqué, invite à voir dans la pièce $\mathrm{A}$ un triclinium ou viridarium. du moins une salle à vocaltion de réception comme le laisse présumer la richesse de sa décoration.

Reste posée lat question de l'accès à cette pièce, qu'il est exclu d'imaginer. au vu des élévations ou des plinthes d'enduits peints encore en place. au nord et à l'est, ni même dans la partie nord du mur occidental.

La restitution d'une ouverture demeure donc envisageable au niveau du mur sud M VII, très arasé. ainsi que dans la partie sud du mur M II. lui aussi bien dégradé, mais dont la maçonnerie semble par contre former un semblant de piédroit à $1.50 \mathrm{~m}$ de l'angle sudouest de la pièce ${ }^{24}$. Ce pourrait être là l'emplacement de la porte. même si cette situation. latérale et complètement décentrée, qui offre en outre au visiteur une vision inversée de l'emblema de la mosaïque. peut paraître surprenante. Cette disposition est fréquente dans l'Antiquité et se rencontre dans la maison au Dauphin à Vaison-la-Romaine (Goudineau, de Kisch. 1991, p. 23). ainsi que dans plusieurs maisons à Pompei : celles dites

23 On le retrouve. sous une forme analogue dans la pièce 1 de l'enclos des Chartreux (voir la contribution d'Alix Barbet dans Bérard ét alii. 1990), p. 25), dans la pièce XV (murs nord el sud) des thermes sud de Notre-Dame d'Avinionet a Mandelieu (voir la contribution de Chantal Maigret dans Fïxo1. 1986, p. 106-111, fig. 6.3. pl. II. 1). dans la pièce 5 de la fouille de la place de la Haute Vieille Tour a Rouen (Catalogue, 1982, n"34, p. 108. lig. 79), ainsi qu'à Boult-surSuippe à Reims (Allage ct alii. 1988. lig. 4. 5.8).

24 Un hiatus de $1.30 \mathrm{~m}$ a élé observé dans la fondation du mur VII. Dans le mur 11 on constate une ligne d'arrêt très nette dans l'appareii qui, à l'inverse du reste de la maçonnerie. emploie ici de gros moellons. «dei Cei», «del Labirinto», "dell Ara massima» 25 entre autres. Laccès à tous ces triclinia s'effectue par une porte latérale.

Dans le cas qui nous occupe, on peut supposer que c'est la fonction même de la pièce qui déterminait toute considération esthétique: sans doute voulait-on privilégier le point de vue offert depuis la banquette plutôt que celui que l'on découvrait depuis l'entrée. Enfin. l'hypothèse d'une ouverture à cet endroit trouve une justification supplémentaire dans le principe de distribution de la maison. Il est logique en effet de penser que l'espace D, qui faisait office de grand vestibule. desservait aussi lat pièce A. Nous n'excluons pas cependant que celle-ci était également accessible par le couloir E. grâce à une porte ménagée dans le mur VII.

Les enduits peints de la pièce A. trouvés disséminés en fragments. effondrés sous forme de plaques, ou en place au pied des murs, forment le lot le plus important des peintures découvertes dans la maison.

Ceux du mur I, trop dégradés, ou trop lacunaires. n'offrent plus guère d'intérêt, mais ceux des murs X et II sont en revanche suffisamment conservés pour que l'on puisse en lire le décor. Subsistent une sous-plinthe d'environ $0.10 \mathrm{~m}$ de haut. de couleur claire. surmontée d'une plinthe à champ noir où court un fin méandre à redans rectangulaire peint en blanc, dans les intervalles duquel prennent place des rectangles limités par un filet ocre-jaune. A l'intérieur de ces demiers. s'inscrivent des fleurons de couleur blanche (fig. 13. p. 201). L'ensemble de cette zone basse est limité par un large filet blanc atl-dessus duquel s'amorce une bande de couleur claire très détériorée. de largeur inconnue.

Des grands panneaux qui devaient constituer la partie moyenne du décor. seuls quelques éléments ont été

25 Voir respectivement: D). Minhl. (asa dei Cei. Haïser in Pompeji. 3. 1990). Munich. Hirmer Verlag. (Deutsches archäologisches Institut. Berlin). pl. 48 : - V.M. Sikock1. Casal del Labirinto, Haïser in Pompejii. 4. 1991.

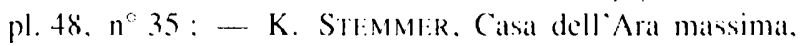
Häiser in Pomperji, 6. 1992. pl. 42. (i. 
retrouvés. Il s'agit de plaques à champ bleu égyptienº qui pourraient provenir de la partic haute des parois. C'est ce que laisse envisager le passage marqué. visible entre le champ bleu et une bande de couleur claire très abîmée, qui correspond peut-être à l'arrachement de stucs. Un nombre restreint de fragments portait, en outre, des traces de couleur ocre-rouge appartenant à des bandes de séparation ou à d'autres motifs inconnus du décor.

Malgré cette restitution incomplète, il y a tout lieu de croire que la décoration picturale de la pièce était asse\% luxueuse à en juger par l'emploi important du bleu égyptien, pigment artificiel assez élaboré et plus coûteux que les pigments naturels (Barbet. 1990). p. 256), qui ne paraît pas avoir été limité aux seules bandes de séparation entre les panneaux, comme c'est le cas, par exemple. dans la domus du parking Pasteur a Aix-en-Provence (Barbet. 1986, p. 31, 35, fig. 51-52). Bien au contraire, cette couleur semble avoir ici constitué le champ des panncaux eux-mêmes.

Le motif qui se développe en zone basse rappelle le décor de l'une des pièces de cette habitation, la salle 5. où l'on retrouve également, mais sur un champ ocrejaune, une ligne de méandres à redans avec carrés inscrits rehaussés au centre d'un fleuron (Barbet. 1991, p. 86. $n^{\circ} 4.3 \mathrm{C}$ ).

Au nord et à l'est de la pièce $A$ ont été très partiellement repérés, sur moins de $1 \mathrm{~m}^{2}$, des vestiges antiques dont l'emprise et l'organisation restent énigmatiques, mais qui semblent toutefois appartenir à un seul espace, l'espace $M$ (fig. 3 et 11). Celui-ci ne pouvait apparemment pas communiquer directement avec la salle $A$ dont les murs mitoyens étaient dépourvus d'ouverture. Nous n'en comnaissons que le sol, constitué de fines dalles calcaires $(0,02 \mathrm{~m}$ d'épaisseur) disposés en quatre strates sur lesquelles a été maçonné un solin de béton de tuileau en quart de rond qui courait le long des murs (fïg. 11). Au nord. est conservé un cinquième rang de dalles qui s'appuie contre ce solin et en masque la base.

L'ensemble de ce dispositif suggère deux interprétations : on peut y voir un aménagement de fond de bassin dont on comprendrait mal, cependant. la configuration en $\mathrm{L}$ et la position dominante par rapport au sol de la piece A. établi $0.60 \mathrm{~m}$ plus bas. Nous y verrions plus volontiers l'amorce d'un espace dallé. tel une cour ou un portique, où l'on aurait pris soin de construire un solin à la base des murs, pour éviter les infiltrations d'eaux dans la pièce attenante.

En l'état des recherches, la différence observée entre les niveaux de sol de ces deux espaces ne trouve pas d'explication immédiate sinon dans une possible adaptation à la topographie du site, ou encore, dans leur appartenance à deux maisons distinctes.

26 Nous devons l'identification de ces pigments au bleu égyptien au Laboratoire de Thiais (CNRS) è à l'amabilité de $\mathrm{M}^{\mathrm{me}}$ Barbet et Coupy que nous remercions.

\section{Datation}

Nous disposons de bien peu d'éléments pour dater la construction et la durée d'occupation de ce bâtiment. où les aménagements primitifs, à peine modifiés dans la pièce $A$ (banquette XVII) semblent avoir perduré jusqu'à son abandon. Les seules informations que nous possédons proviennent de l'analyse stylistique du décor de la pièce A ainsi que de l'étude des céramiques issues du sol en terre de la pièce $H$ et de ses niveaux de préparation 27 .

La présence de céramiques culinaires africaines dans ces remblais situe leur installation après la fin du $l^{e r} s$. de notre ère. Le sol recelait quant à lui une sigillée claire $\mathrm{B}$ de la forme Desbat 19 (14()-210), une sigillée claire A de la forme Hayes $3(60-140)$ et des céramiques culinaires africaines Hayes 23, 196 et 197. qui placent son utilisation au plus tôt vers le milieu du II's. Celleci dura au moins jusqu'à la fin du siecle comme l'atteste la découverte d'une sigillée claire B de la forme Desbat $53(170-19(0)$, trouvée écrasée à la surface du sol.

Si la datation que propose $\mathrm{H}$. Lavagne (infra. p. 21.3) pour la mosaïque de la pièce $A$ nous renvoie également vers le milieu du II ${ }^{\mathrm{S}}$ s.. celle du décor mural qui l'accompagne peut sembler à première vue nettement plus ancienne. La peinture de la partie basse du mur X. qui repose sur le pavement. relève en effet du III ${ }^{\circ}$ ou du IV' style pompéien dont le terminus ante quem est les années 90 de notre ère (Barbet, 1985, p. 273). Dans le cas présent, il nous paraît cependant plus vraisemblable de penser que ce schéma était encore utilisé dans la peinture provinciale au cours du $\mathrm{II}^{\mathrm{C}} \mathrm{s}$.

Le décor d'imitation de marbre à grandes ondulations de la banquette XVII est en revanche bien caractéristique de la peinture du $I^{\mathrm{C}} \mathrm{s}$ : les exemples analogues rencontrés à Aix-en-Provence. Mandelieu et Rouen sont datés de la première moitié de ce siècle et celui de Boultsur-Suippe de la seconde.

En dépit de l'absence de tout élément chronologique dans les autres pièces du bâtiment. l'homogénéité de la construction suggère de faire remonter l'implantation de cet ensemble vers le milieu du $\mathrm{II}^{\mathrm{C}} \mathrm{s}$.

La date de son abandon, est difficile à préciser. les niveaux de destruction, en partie remaniés, n'ayant livré qu'un matériel atypique ou contemporain de l'occupation, à l'exception de trois tessons de D.S.P. 28 auxquels ne sont associés ni sol, ni construction.

Faut-il envisager pour autant que nous avons là un exemple précoce d'abandon de maison urbaine, ou plutôt que les traces éventuelles d'une occupation plus tardive aient disparu lors de la construction des maisons médiévales?

27 Aucun matériel n'a été trouvé dans les radiers des sols des autres espaces.

28 Liun des trois fragments est un bord de la forme Rigoir 18. daté fin IVe-Vés dans les fouilles de Marseille (Cavaillès, Llopis, 1986, p. 170). 
A défaut de connaître l'organisation générale des bâtiments, il est difficile de préciser s'ils appartenaient à une ou deux habitations, bien que l'existence possible d'une rue à l'emplacement de l'espace I nous amène à privilégier la seconde hypothèse.

Quoi qu'il en soịt, et bien qu'un certain nombre d'autres questions restent posées, les recherches menées sur le site des Magnans enrichissent nos connaissances sur la topographie antique d'Aix-en-Provence, car elles permettent d'entrevoir la nature de l'urbanisation dans un quartier proche du centre monumental de la cité.

La présence de mosaïques, polychromes ou non, d'enduits peints aux pigments coûteux ou d'aménagements soignés, tel le puits de l'espace $\mathrm{K}$, rend compte de la qualité de ces installations.

Bien différents des insulae nettement plus modestes découvertes aux abords du fortum, situé à moins de $300 \mathrm{~m}$, ces vestiges d'habitations sont en revanche tout à fait comparables à ceux des riches domus mises au jour au nord et à l'ouest de la ville antique (maisons du parking Pasteur (Boiron et alii, 1986), du jardin de Grassi (Benoît, 1947), des enclos Milhaud et Reynaud (Rouard, 1941 et 1943), ou de la rue des Chartreux (Bérard et alii. 1990). Il est d'ailleurs frappant de constater qu'un même atelier de mosaïstes a réalisé le pavement de la rue des Magnans, et ceux, à motif identique, de deux de ces demeures aixoises (Lavagne, 1990, p. 20-23).

La seule différence notable entre certaines de ces maisons et les bâtiments découverts ici concerne la taille des pièces, qui semble, comme sur le site des Chartreux, relativement modeste si on la compare à la superficie des grandes salles (plus de $80 \mathrm{~m}^{2}$ ) des domus des quartiers nord. Cette constatation, basée sur une vision très lacunaire du bâtiment supérieur, doit cependant être relativisée.

Le lotissement du site des Magnans, vers le milieu du II $^{\mathrm{e}} \mathrm{s}$., apparaît assez tardif si l'on considère que le coeur de la ville ${ }^{29}$ (Fixot et alli, 1986, p. 198-204) et les quartiers ouest (Bérard et alii, 1990, p. 11-12: Kauffmann, 1983) étaient urbanisés, ou en voie de l'être. à l'époque augustéenne. Il est en outre postérieur à la grande période de développement urbain qui, à la fin du Ios s. de notre ère, voit l'achèvement de l'ensemble monumental du forum et la transformation du quartier qui l'entoure selon un plan d'urbanisme réglé (Fixot et alli. 1986, p. 204-220).

Faut-il donc envisager que ce site ait pu être occupé avant le milieu du IIC siècle? Si cette hypothèse paraît peu probable pour la terrasse supéricure, la question reste posée pour la terrasse inférieure. où, faute d'avoir fouillé les sols en place, un seul état d'occupation a été mis en évidence.

29 Cf. supra. note 6.
Il est d'autre part essentiel de constater que les bâtiments du site des Magnans adoptent les mêmes orientations que celles du for um. et de l'ensemble monumental découvert sur le site des Thermes, orientations qui se retrouvent également dans certains quartiers périphériques d'Aquae Sexilae.

\section{G. BÉRARD, B. DE LUCA, C. LANDURÉ et A. NICOLAÏDÈS}

\section{Nota Bene -}

Campagne de reconnaissance et suivi administratif : $M$. Bonifay :-campagne de fouilles : C. Landuré, G. Bérard, B. De Luca pour l'encadrement, A. Bougobba, N. Rehala, J.-M. Sabatier et P. Reynaud (bénévole) pour l'équipe de fouille : — sondage dans la rue des Magnans : A. Nicolaïdès et N. Nin avec la participation de C. Socratous, M. Lutti, F. Parent et R. Thernot:- dépose de la mosaique : atelier de restauration de mosaïques de Saint-Romain-en-Gal.

Cette étude a bénéficié du concours technique de P. Chapon pour la mise au net des plans et coupes (fig. 1, 2, 3, 4): C. Hussy, Atelier d'Arts Graphiques, S.R.A. PACA (fig. 6) ; N. Nin, Service Archéologique de la ville d'Aix (fig. 7, 11-13) : F. Parent pour les prises de vues du sondage de la rue des Magnans (fig. 5, 8-10): R. Thernot pour les relevés du sondage de la rue des Magnans (fig. 3, 11) et de la collaboration scientifique de A. Barbet. M. Bonifay, M. Coupy. J. Guyon, H. Lavagne (étude de la mosaïque). N. Mingaud ei N. Nin. Nous remercions également, pour l'aide qu'il nous a apporté. le Service régional de l'Archéologie de Provence-Alpes-Côte d'Azur.

\section{BIBLIOGRAPHIE}

\author{
Allag C. et alii \\ 1988: La mort d'Adonis : une peinture murale gallo-romaine \\ à Boult-sur-Suippe (Marne), Bulletin de la Société \\ archéologique champenoise, 81 (1988) n². p. 93-110. \\ Ambard R. \\ 1984: Aix Romaine. Nouvelles observations sur la topo - \\ graphic d'Aquae Sertiae. Aix-en-Provence, éd. Ass. \\ Archéologique «Entremont», 246 p.. 116 fig. \\ Barbet A. \\ 1985: La peimture murale romaine. Paris, Picard. 285p.. \\ 200 lig. \\ 1986 : La maison et le décor, in : Boiron R., Landuré C., Nin \\ N., Les fomilles de l'aire du Chapitre. coll. Documents \\ d'Archéologie Aixoise, 2, Aix-en-Provence, p. 27-35. \\ 1990: L'emploi des couleurs dans la peinture murale antique : \\ "marqueurs" chronologiques et révélateurs du «standing" \\ social. Pigme'nts e't colorants. Paris. éd. du CNRS, p. 25.5 - \\ 271. \\ 1991: Peintures romaines à Narbonne. Décorations mu- \\ rales de l'antique province de Narbonnaise. Narbonne. éd. \\ DRAC de la région Languedoc-Roussillon. \\ Benoit F. \\ 1947 : La maison à double péristyle du jardin de Cirassi à Aix - \\ en-Provence, Gallia. V. 1. p. 98-122.
}




\section{Bérard G. De Luca B., Landuré C. et alii}

1990: Les fouilles de l'enclos des Chartreux, De l'Antiquité (ar XVII's., coll. Documents d'Archéologie Aixoise, 5, Aixen-Provence, 64 p., 97 fig.

Boiron R., Landuré C., Nin N.

1986: Les fouilles de l'Aire du Chapitre, coll. Documents d'Archéologie Aixoise, 2, Aix-en-Provence, 47 p., 64 fig.

\section{Bonifay M.}

1990: Rapport d'expertise archéologique : 18, rue des Magnans, Aix-en-Prolence, 1 vol. dactyl.

\section{Cavaillès Llopis M.-T.}

1986: Céramiques de l'Antiquité tardive à Marseille, Documents d'Archéologie Méridionale, 9, p. 167-195.

\section{Desbat A.}

1980: Les céramiques fines rhodaniennes à vernis argileux dites sigillées claires $B$ et luisantes. Etude du matériel lyonnais des $I^{\prime}$ et $I I I^{\circ}$ s., thèse de $3^{\mathrm{e}}$ cycle. Univ. de Lyon.

Fixot M., Guyon J., Pelletier J.-P., Rivet L.

1986: Des abords du forum au palais archiépiscopal - Etude du centre monumental d'Aix-en-Provence, Bulletin Monumental. 144, III, p. 196-290.

Goudineau C., de Kisch Y.

1991 : Vaison-la-Romaine, Paris, éd. Errance, 159 p.

Hayes J. W.

1972 : Late Roman Pottery, Londres, 477 p., 93 fig., $23 \mathrm{pl}$. . 40 cartes.

\section{Kauffmann A}

1983: Aix-en-Provence antique. Topographie et érolution urhaine, Mémoire de maîtrise, Aix-en-Provence, Univ. de Provence.

\section{Lavagne $\mathrm{H}$.}

1990 : Les mosaïques, in : Bérard G., De Luca B., Landuré C., Les fouilles de l'enclos des Chartreux, De l'Antiquité au $X V I I^{\prime}$ s., coll. Documents d'Archéologie Aixoise, 5, p. 20-23. Maigret $\mathbf{C}$.

1990 : Le décor des pièces d'entrée des thermes sud, in : Fixot M. (dir.), Le site de Notre-Dame d'Avinionet à Mandelieu. Monographie du CRA n ${ }^{\circ}$ 3, Paris, éd. du CNRS, p. 104-141. Nicolaïdès A., Nin N.

1992 : Rue des Magnans. Rapport de sauvetage urgent. I vol. dactyl.

Nin $\mathbf{N}$.

1990 : Rues Gibelin et de la Louvière, Gallia Informations, 1-2, p. 136-137.

La peinture murale romaine...

1982: La peinture murale romaine de la Picardie à la Normandie. Catalogue d'exposition 1982-1984, Rouen, ód. CNRS.

Rigoir J., Rigoir Y.

1985: Dérivées des sigillées paléochrétiennes provençales, Catalogue des formes, Paris.

\section{Rouard E.}

1841 et 1943 : Rapports sur les fouilles des antiquités qui ont été faites à Aix, Aix-en-Provence, Commission d'Archéologie d'Aix.

\section{Vitruve}

De Architectura, traduction F. Granger, éd. W. Heinemann, Cambridge (Massachusetts), 1962, Harvard University Press, Livre VII, vol. 2.

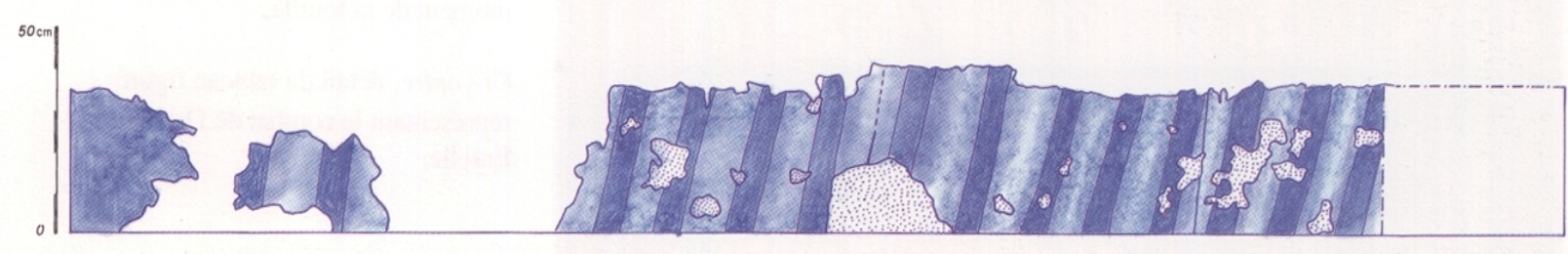

Fig. 12 - Relevé de face de la peinture murale ornant la banquette XVII.

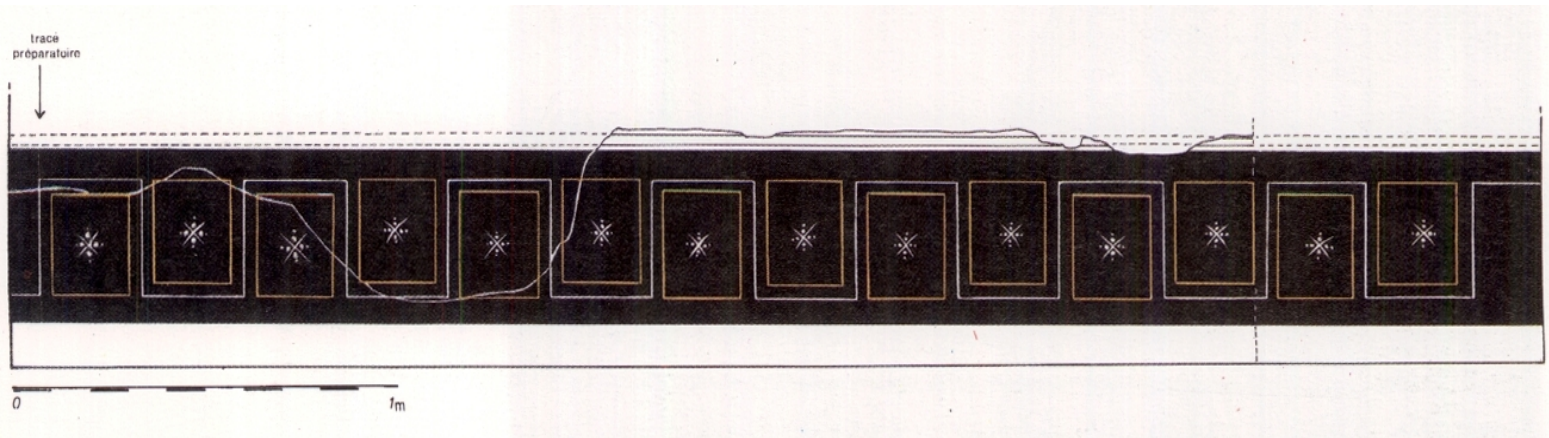

Fig. 13 - Relevé de la peinture murale. 